\title{
The welfare benefits of stable and efficient payment systems
}

\author{
Stephen Millard \\ and \\ Matthew Willison
}

Bank of England, Threadneedle Street, London, EC2R 8AH.

E-mail: stephen.millard@bankofengland.co.uk

matthew.willison@bankofengland.co.uk

The views expressed are those of the authors and do not necessarily reflect those of the Bank of England.

Copies of working papers may be obtained from Publications Group, Bank of England, Threadneedle Street, London, EC2R 8AH; telephone 0207601 4030, fax 0207601 3298, e-mail mapublications@bankofengland.co.uk

Working papers are also available at www.bankofengland.co.uk/wp/index.html

The Bank of England's working paper series is externally refereed.

C Bank of England 2003

ISSN 1368-5562 



\section{Contents}

$\begin{array}{ll}\text { Abstract } & 5\end{array}$

$\begin{array}{lr}\text { Summary } & 7\end{array}$

$1 \quad$ Introduction and overview 9

$\begin{array}{lll}2 & \text { The basic model } & 10\end{array}$

$\begin{array}{ll}3 & 12\end{array}$

$4 \quad$ Operational risk 15

$5 \quad$ Welfare 18

$\begin{array}{lll}5 & \text { Conclusions } & 20\end{array}$

References 



\begin{abstract}
In this paper, we develop a theoretical model of the introduction of a payment system into an economy in which transactions had previously been carried out using cash. We then use this model to measure the welfare benefits of such a change in terms of the model's parameters. We find that if the cost of using the payment system is low enough and that the payment system is sufficiently reliable, then agents prefer there to be a payment system they can use rather than have to carry money around with them. Furthermore, we find that social welfare is unambiguously decreasing in the cost of using the system. We find that a decrease in the reliability of the payment system is also welfare reducing.
\end{abstract}




\section{Summary}

To be written 
The Bank of England's second core purpose is to maintain the stability of the financial system, both domestic and international. A recent speech by Padoa-Schioppa (2002) defined financial stability as 'a condition where the financial system is able to withstand shocks without giving way to cumulative processes which impairs (sic) the allocation of savings to investment opportunities and the processing of payments in the economy'. The purpose of this paper is to concentrate on the latter part of that definition - viz. the payment systems aspects of financial stability - since it is clear that a smoothly functioning payments system is a necessary condition for financial stability and, so, it will be important, to know how to measure the benefits of this.

In particular, we consider the following definition of financial stability in a payment systems context: a stable payment system is one in which shocks emanating from or propagated by users of the system or the system itself do not compromise agents ability to transact by their chosen means. Examples of 'shocks emanating from users of the system' could include bankruptcy of participants, liquidity shortages among participants or problems with individual banks operations. Examples of 'shocks propagated by users of the system' could include productivity, preference or government spending surprises that lead to changes in the profitability or liquidity holdings of participants in the system. And, finally, examples of 'shocks to the system itself' could include operational problems leading to a temporary closure of the system. This definition acknowledges that a shock to the payment system may not necessarily affect consumers' aggregate savings ratio but would still reduce welfare in this case by reducing the set of goods that 'variety-loving' consumers were able to consume, since their ability to consume certain goods may be affected by the means of payment that they can/cannot use. This, we think, captures the essence of what makes a 'payment system' different to other aspects of the financial system.

He et al (2003) present a search theoretic model of a payment system. In their model agents have a choice between two means of making payments but only one, cash, can be stolen. They refer to the safe alternative to cash as cheques. Agents can deposit their cash in banks and the banks issue them with cheque books. But the use of cheques requires there to be an inter-bank payment system. The authors find that the introduction of cheques (and banks) expands the range of parameter values consistent with there being an equilibrium in which money is accepted as a medium of exchange.

But the payment system in He et al (2003) is risk free. Lester (2004) extends the He et al (2003) model to incorporate the potential for banks to go bankrupt and hence, default on their payment obligations. When a producer receives a cheque he is in effect extending credit to the buyer until the cheque is settled. But during the lag events may take place that mean that the buyer's bank does not have enough money when the cheque is due to be settled. That is, there is credit risk. Lester assumes that banks can default during the lag because they are hot by some exogenous shock. As banks hold fractional reserves, a holder of a cheque may find that he does not receive any money for a good that he has 
sold. Lester recalculates the conditions under which agents use cheques in the presence of credit risk.

This paper considers how shocks to the payment system itself affect agents' incentives to use the system in the He et al (2003) model. When a buyer and a producer meet and agree to trade, the payment system only functions with some probability. We will refer to this risk as operational risk. The fact that the payment system fails before a payment is initiated implies that we are not thinking about cheques in our story but instead a means of payment, such as debit card payments, which requires the sending of an electronic message at the point of sale. We show that agents have an incentive to use the payment system if the system is sufficiently cheap to use and/or it is sufficiently reliable and derive lower bounds on the probability that the payment system functions (conditional on the cost of using it) that are consistent with buyers choosing to use the payment system. We do so for two cases: (1) when money would not be accepted as a medium of exchange in the absence of the payment system; (2) when money would be accepted in the absence of the payment system. Finally, we compare social welfare with and without the payment system for each of the cases. The introduction of a payment system that buyers and producers choose to use unambiguously increases social welfare if it expands the number of trades occurring in the economy, that is, makes money an accepted medium of exchange. When money is accepted as a medium of exchange in the absence of a payment system, social welfare can increase or decrease with the introduction of a payment system. In this case, the addition of a payment system will not expand the number of trades that occur in our model; so there will be no social benefit arising from this channel. Social welfare will only increase if the reduction in deadweight loss caused by theft in the economy (modelled as a cost that thieves incur when they rob successfully) is sufficiently greater than the costs of using the payment system (including both the direct costs of using the system and the costs related to when the system fails).

The basic He et al (2003) model is introduced in Sections 2 and 3. We analyse a payment system that suffers from operational risk in Section 4. A social welfare comparison is performed in Section 5. Section 6 concludes.

\section{The basic model}

In this section we will sketch the version of the He et al (2003) model where a move by nature in each period determines whether an agent that does not hold money is a producer or a thief. (He et al refer to this case as one of 'exogenous robbery'.) The model is builds on the Lagos-Wright (2002) framework. Each period is comprised of two subperiods. Centralised trade occurs in one sub-period. Decentralised trade, involving search frictions, takes place in the other sub-period. In other variants of the model the existence of the two sub-periods is important. However, this is not true in this paper and we shall proceed without discussing the centralised market. 
The economy consists of a unit continuum of agents. We assume that there is a measure, $m$, of agents holding one unit of money each. We refer to these agents as buyers. A buyer receives utility, $u$, each time he consumes a good he wants. Money is indivisible. The remaining agents either are thieves or producers, the proportion of thieves (producers) being $\lambda(1-\lambda)$. A producer can produce one unit of a specialised, divisible good at a cost $c$. We assume that trade is worthwhile, i.e., $u>c$. One good trades for one unit of money. In a meeting between a buyer and a producer, the buyer wants the producer's good with probability $x$. Barter (between two producers) occurs with probability zero. In a meeting between a buyer and a thief, the thief attempts to steal the buyer's unit of money. He is successful with probability $\gamma$. If he successfully robs a buyer he incurs a cost $z$.

Let $V_{m}$ and $V_{0}$ be the values of being a buyer and a non-buyer, respectively. Agents have a discount rate $r$. Agents' respective Bellman equations are shown in equations (1) and (2).

$$
\begin{aligned}
& r V_{m}=x(1-m)(1-\lambda)\left[u+V_{0}-V_{m}\right]+\gamma \lambda(1-m)\left[V_{0}-V_{m}\right] \\
& r V_{0}=x m(1-\lambda)\left[V_{m}-V_{0}-c\right]+\gamma \lambda m\left[V_{m}-V_{0}-z\right]
\end{aligned}
$$

Trade occurs in a monetary equilibrium; i.e., an equilibrium in which money is an accepted medium of exchange. The conditions that must be met in any monetary equilibrium are: $V_{m} \geq 0, V_{0} \geq 0$, and $V_{m}-V_{0} \geq c$. The first two conditions are participation conditions. These must be satisfied for both buyers and non-buyers to prefer to be active in the economy rather than drop out and live in autarchy. The third condition is an incentive condition. It ensures that when a producer meets a buyer who wants his good, the producer has an incentive to accept a unit of money as payment. Following $\mathrm{He}$ et al, we assume that a thief steals irrespective of whether his payoff from successfully robbing an agent, $V_{m}-V_{0}$, exceeds his cost from doing so, $z$. We need only check that $V_{0} \geq 0$ and $V_{m}-V_{0} \geq c$ because when these conditions both hold it must be that $V_{m} \geq 0$.

\section{Proposition 1}

There exists a monetary equilibrium if and only if

$$
\begin{aligned}
& \frac{x(1-m)(1-\lambda) u+\gamma \lambda z}{r+x(1-m)(1-\lambda)+\gamma \lambda} \geq c \text { and } \\
& \frac{(1-m)[x(1-\lambda)+\gamma \lambda]}{r+(1-m)[x(1-\lambda)+\gamma \lambda]} u-\frac{\gamma \lambda}{x(1-\lambda)} z \geq c
\end{aligned}
$$




\section{Proof}

If we subtract $r V_{0}$ from $r V_{m}$ and rearrange, we find

$$
\begin{gathered}
V_{m}-V_{0}=\frac{x(1-m)(1-\lambda) u+x m(1-\lambda) c+\gamma \lambda m z}{r+x(1-\lambda)+\gamma \lambda} \\
V_{m}-V_{0} \geq c \Leftrightarrow \begin{array}{c}
x(1-m)(1-\lambda) u+x m(1-\lambda) c+\gamma \lambda m z \geq[r+x(1-\lambda)+\gamma \lambda] c \\
\frac{x(1-m)(1-\lambda) u+\gamma \lambda m z}{r+x(1-m)(1-\lambda)+\gamma \lambda} \geq c
\end{array}
\end{gathered}
$$

$V_{0} \geq 0 \Leftrightarrow r V_{0} \geq 0$

$$
V_{m}-V_{0} \geq \frac{x m(1-\lambda) c+\gamma \lambda m z}{x m(1-\lambda)+\gamma \lambda m}
$$

If we substitute the above expression for $V_{m}-V_{0}$ and rearrange, we find

$V_{0} \geq 0 \Leftrightarrow \frac{(1-m)[x(1-\lambda)+\gamma \lambda]}{r+(1-m)[x(1-\lambda)+\gamma \lambda]} u-\frac{\gamma \lambda}{x(1-\lambda)} z \geq c$

Proposition 1 is the same as proposition 1 in He et al. It shows that a producer will accept one unit of money as payment for its good if the utility from consuming, $u$ is sufficiently greater than the production cost, $C$.

\section{Adding a payment system}

We will now introduce a technology that enables money to flow between buyers and producers without buyers having to carry money around on their persons: that is, a payment system. A buyer deposits his money into a bank and is issued with a cheque book or a debit card. If a buyer carries money on his person we say that he uses cash. The key advantage of using the payment system, rather than cash, is that it is immune from the risk of robbery. Payments flow between banks in the centralised market that we have up until now subsumed. Banks are subject to $100 \%$ reserve requirements. Banks do not issue loans. As in $\mathrm{He}$ et al, we assume that banks settle their payment obligations without fail. (Lester (2004) relaxes this assumption by introducing bank defaults into the He et al model when banks have less than $100 \%$ reserve requirements.) Finally, if a buyer uses the payment system, he is charged a price, $p(\geq 0)$.

Let $\theta$ be the measure of buyers who use the payment system. The Bellman equations for when there is a payment system are shown in equations (3)-(4). 
$r V_{m}=\max \left\{\begin{array}{c}x(1-m)(1-\lambda)\left(u+V_{0}-p-V_{m}\right), \\ x(1-m)(1-\lambda)\left(u+V_{0}-V_{m}\right)+\gamma \lambda(1-m)\left(V_{0}-V_{m}\right)\end{array}\right\}$

(3)

$r V_{0}=x m(1-\lambda)\left(V_{m}-V_{0}-c\right)+\gamma \lambda m(1-\theta)\left(V_{m}-V_{0}-z\right)$

Consider an equilibrium in which all agents use the payment system. In this case, it must be the case that

$x(1-m)(1-\lambda)\left(u+V_{0}-p-V_{m}\right) \geq x(1-m)(1-\lambda)\left(u+V_{0}-V_{m}\right)+\gamma \lambda(1-m)\left(V_{0}-V_{m}\right)$

where

$r V_{m}=x(1-m)(1-\lambda)\left(u+V_{0}-p-V_{m}\right)$

$r V_{0}=x m(1-\lambda)\left(V_{m}-V_{0}-c\right)$

The equilibrium conditions are: $V_{m} \geq 0, V_{0} \geq 0$, and $V_{m}-V_{0} \geq c . V_{m}-V_{0} \geq c$ implies $V_{0} \geq 0$ and, hence, $V_{m} \geq 0$. Thus, we need only derive the conditions under which $V_{m}-V_{0} \geq c$ and equation (5) holds.

If we subtract $r V_{0}$ from $r V_{m}$ we find

$V_{m}-V_{0}=\frac{x(1-m)(1-\lambda)(u-p)-x m(1-\lambda) c}{r+x(1-\lambda)}$

Hence, $V_{m}-V_{0} \geq c$ if

$x(1-m)(1-\lambda)(u-p)-x m(1-\lambda) c \geq[r+x(1-\lambda)] c$

that is

$(u-p) \frac{x(1-m)(1-\lambda)}{r+x(1-m)(1-\lambda)} \geq c$ 
Rearranging equation (5) implies

$\frac{\gamma \lambda\left(V_{m}-V_{0}\right)}{x(1-\lambda)} \geq p$

If we substitute the expression for $V_{m}-V_{0}$ into equation (11) and rearrange we obtain:

$\frac{(1-m)}{m} u-\frac{r+x(1-\lambda)+\gamma \lambda(1-m)}{\gamma \lambda m} p \geq c$

If we rearrange equations (10) and (12) so that $p$ is on the left-hand side, we find the conditions that $p$ must satisfy for buyers to have an incentive to use the payment system. These are shown in equations (14) and (15), respectively.

$$
\begin{aligned}
& p_{1}=u-\frac{r+x(1-m)(1-\lambda)}{x(1-m)(1-\lambda)} c \geq p \\
& p_{2}=\frac{(1-m) \gamma \lambda}{r+x(1-\lambda)+\gamma \lambda(1-m)} u-\frac{\gamma \lambda m}{r+x(1-\lambda)+\gamma \lambda(1-m)} c \geq p
\end{aligned}
$$

Combining equations (13) and (14) and the fact $p$ must be non-negative, we get the condition in equation (15):

$\min \left\{p_{1}, p_{2}\right\} \geq p \geq 0$

We have the result that buyers will choose to use the payment system if it is sufficiently cheap to do so. Equation (15) can hold when there is not a monetary equilibrium in the absence of a payment system and when there is. Both $p_{1}$ and $p_{2}$ are increasing in $u$. That is, the more utility a buyer gets from consumption, the more he is prepared to pay for using the payment system. $p_{1}$ and $p_{2}$ are decreasing in $c$ since a higher production cost reduces the gains from becoming a producer (following consumption) and hence, a buyer will only use the payment system if the price is lower. When a buyer faces a high probability of being robbed, he is willing to pay a higher price for using the payment system. Both $p_{1}$ and $p_{2}$ are increasing in $\lambda$, showing that buyers are willing to pay 
more for the payment system the greater is the probability of being robbed. ${ }^{1}$ The price a buyer is ready to pay is also increasing in the probability that an attempted robbery is successful, $\gamma$, if $p_{2} \geq p_{1}{ }^{2}$

\section{$4 \quad$ Operational risk}

So far, we have considered a payment system that functions without fail. In the real world, payment systems are susceptible to operational events that prevent agents from using it. Introducing operational events into the model is relatively straightforward. We simply assume that such events are Poisson. In particular, we assume that the arrival rate of the system not functioning is $1-\delta$. The Bellman equations are shown in equations (16)-(17).

$$
\begin{aligned}
& r V_{m}=\max \left\{\begin{array}{c}
\delta x(1-m)(1-\lambda)\left[u+V_{0}-p-V_{m}\right], \\
x(1-m)(1-\lambda)\left[u+V_{0}-V_{m}\right]+\gamma \lambda(1-m)\left[V_{0}-V_{m}\right]
\end{array}\right\} \\
& r V_{0}=x m(1-\lambda)(1-\theta(1-\delta))\left(V_{m}-V_{0}-c\right)+\gamma m \lambda(1-\theta)\left(V_{m}-V_{0}-z\right)
\end{aligned}
$$

In an equilibrium in which agents are using the payment system (i.e., $\theta=1$ ), the Bellman equations become:

$$
\begin{aligned}
& r V_{m}=\delta x(1-m)(1-\lambda)\left(u+V_{0}-p-V_{m}\right) \\
& r V_{0}=\delta x m(1-\lambda)\left(V_{m}-V_{0}-c\right)
\end{aligned}
$$

The conditions that must hold for $\theta=1$ in equilibrium are:

$$
V_{m}-V_{0} \geq c
$$$$
\delta x(1-m)(1-\lambda)\left(u+V_{0}-p-V_{m}\right) \geq x(1-m)(1-\lambda)\left(u+V_{0}-V_{m}\right)+\gamma \lambda(1-m)\left(V_{0}-V_{m}\right)
$$

\footnotetext{
${ }^{1}$ In fact, $\partial p_{2} / \partial \lambda \geq 0$ if and only if $\gamma \lambda(1-m) u \geq \gamma \lambda m c$. However, this inequality will hold if $p_{2} \geq 0$.
} 
If we follow the same procedure as before, we find that the payment system will be used if and only if

$$
\min \left\{\begin{array}{l}
u-\frac{r+\delta x(1-m)(1-\lambda)}{\delta x(1-m)(1-\lambda)} c, \frac{-r(1-\delta)-\delta x m(1-\lambda)(1-\delta)+\lambda(1-m)}{\delta[r+x(1-\lambda)-x m(1-\lambda)(1-\delta)+\lambda(1-m)]} u \\
+\frac{m[x(1-\lambda)(1-\delta)+\lambda]}{r+x(1-\lambda)-x m(1-\lambda)(1-\delta)+\lambda(1-m)} c
\end{array}\right\} \geq p
$$

In addition, the cost of making a payment $p$ must be non-negative. Thus, we can derive conditions that the reliability of the payment system, $\delta$, must satisfy for equation (20) and $p \geq 0$ to be met. That is, lower bounds on the reliability of the payment system consistent with agents having an incentive to use the payment system given the price for doing so is non-negative (i.e., they are not being subsidised for using the system). For simplicity, we will assume $z=0$. If we apply the restriction $p \geq 0$ to equation (20) and rearrange we obtain equation (21).

$u-\frac{r+\delta x(1-m)(1-\lambda)}{\delta x(1-m)(1-\lambda)} c \geq 0 \geq u-\frac{\delta m[x(1-\lambda)(1-\delta)+\lambda]}{r(1-\delta)+\delta x m(1-\lambda)(1-\delta)-\lambda(1-m)} c$

We will now derive lower bounds on values of $\delta$ consistent with equation (21):

$u-\frac{r+\delta x(1-m)(1-\lambda)}{\delta x(1-m)(1-\lambda)} c \geq 0 \quad \Leftrightarrow \quad \delta \geq \frac{r c}{x(1-m)(1-\lambda)(u-c)}=\delta_{1}$

$0 \geq u-\frac{\delta m[x(1-\lambda)(1-\delta)+\lambda]}{r(1-\delta)+\delta x m(1-\lambda)(1-\delta)-\lambda(1-m)} c \Leftrightarrow \delta \geq \delta_{2}$

where $\delta_{2}{ }^{2} x m(1-\lambda)(u-c)+\delta_{2}[r u+\lambda m c-x m(1-\lambda)(u-c)]-[r-\lambda(1-m)] u=0$.

The next step is to derive conditions on $\delta$ that must hold for equation (21) to be consistent with whether or not there is a monetary equilibrium in the absence of the payment system. Equation (22) must hold if there is not a monetary equilibrium when there is not a payment system.

\footnotetext{
${ }^{2}$ To get this result we once again appeal to the fact $\gamma \lambda(1-m) u \geq \gamma \lambda m c$.
} 
$u-\frac{r+\delta x(1-m)(1-\lambda)}{\delta x(1-m)(1-\lambda)} c \geq 0 \geq u-\frac{r+x(1-m)(1-\lambda)+\gamma \lambda}{x(1-m)(1-\lambda)} c$

Equation (22) can hold if and only if

$\delta \geq \frac{r}{r+\gamma \lambda}=\delta_{3}$

Equation (23) must hold if there is a monetary equilibrium when there is not a payment system.

$u-\frac{r+x(1-m)(1-\lambda)+\gamma \lambda}{x(1-m)(1-\lambda)} c \geq 0 \geq u-\frac{\delta m[x(1-\lambda)(1-\delta)+\lambda]}{r(1-\delta)+\delta x m(1-\lambda)(1-\delta)-\lambda(1-m)} c$

Equation (23) can hold if and only if

$\delta \geq \delta_{4}$

where

$\delta_{4}{ }^{2} x m(1-\lambda)(r+\gamma \lambda)+\delta_{4}(\lambda x(1-m)(1-\lambda)-x m(1-\lambda)(r+\gamma \lambda)-r[r+x(1-m)(1-\lambda)+\gamma \lambda])$

$-(r-\lambda(1-m))[r+x(1-m)(1-\lambda)+\gamma \lambda]=0$

We summarise these results in Proposition 2.

\section{Proposition 2}

(i) If there is not a monetary equilibrium in the absence of a payment system, there exists a feasible payment system that buyers use if $1 \geq \delta \geq \max \left\{0, \delta_{1}, \delta_{2}, \delta_{3}\right\}$. 
(ii) If there was a monetary equilibrium in the absence of a payment system, there exists a feasible payment system that buyers use if $1 \geq \delta \geq \max \left\{0, \delta_{1}, \delta_{2}, \delta_{4}\right\}$.

\section{$5 \quad$ Welfare}

We will now consider whether the introduction of a payment system increases social welfare. Let $S W$ be the level of social welfare in a monetary equilibrium when there is no payment system and $S W_{\mathrm{p}}$ be the level of social welfare when there is a payment system and it is used. Suppose that the arrival rate of operational problems that cause the payment system to fail to function is $1-\delta$. The social welfare functions (multiplied by the discount rate) are shown in equations (24)-(26). Equation (24) is the social welfare function when there is not a monetary equilibrium in the absence of a payment system. It equals zero because there is no trade. Equation (25) is the social welfare function when there is a monetary equilibrium even when there is not a payment system.

$$
\begin{array}{rlrl}
r S W & =m r V_{m}+(1-m) r V_{0} & \text { if } \frac{x(1-m)(1-\lambda) u+\gamma \lambda m z}{r+x(1-m)(1-\lambda)+\gamma \lambda}<c \\
& =0 & \\
r S W & =x m(1-m)(1-\lambda)(u-c)-\gamma \lambda m(1-m) z & \text { if } \frac{x(1-m)(1-\lambda) u+\gamma \lambda m z}{r+x(1-m)(1-\lambda)+\gamma \lambda} \geq c \\
r S W_{p}=\delta x m(1-m)(1-\lambda)(u-p-c) &
\end{array}
$$

Equation (26) shows that in an economy in which a payment system is used, a reduction in the cost of using it, $p$, leads to an increase in social welfare. An increase in the reliability if the payment system, $\delta$, also leads to an increase in social welfare.

Propositions 3 and 4 consider under what conditions the introduction of a payment system increases social welfare.

\section{Proposition 3:}

The introduction of a payment system into a monetary economy leads to an increase in social welfare if there is not a monetary equilibrium in the absence of a payment system.

\section{Proof:}


In an equilibrium in which the payment system is used, $V_{0} \geq 0$ and $V_{m}>0$, and so $S W_{p}>0=S W$.

\section{Proposition 4:}

The introduction of a payment system into a monetary economy leads to an increase in social welfare if there is a monetary equilibrium in the absence of a payment system if and only if

$\frac{\gamma \lambda}{\delta x(1-\lambda)} z-\frac{(1-\delta)}{\delta}(u-c)>p$

\section{Proof:}

From equations (25) and (26)

$$
\begin{aligned}
& r\left(S W_{p}-S W\right)=m(1-m)[\gamma \lambda z-x(1-\lambda)(1-\delta)(u-c)-\delta x(1-\lambda) p]>0 \\
& \Leftrightarrow \frac{\gamma \lambda}{\delta x(1-\lambda)} z-\frac{(1-\delta)}{\delta}(u-c)>p
\end{aligned}
$$

Robbery reduces social welfare because it can deter agents from trading and because thieves incur the cost $z$ when they are successful. If there is not a monetary equilibrium in the absence of a payment system, it is because the threat of robbery is deterring agents from trading. Thus, the introduction of a payment system that agents use will unambiguously increase social welfare since it leads to trade taking place. When there is a monetary equilibrium without a payment system, the introduction of a payment system does not lead to more trade. Thus, one of the channels through the introduction of a payment system can improve social welfare is closed when there is a monetary equilibrium in the absence of a payment system. In this case, the introduction of a payment system can only increase social welfare through the fact that it results in no thief incurring the cost $z$ anymore. But the expected savings in terms of the cost $z$ must exceed the expected costs from using the payment system. The expected cost saving from eliminating robbery occurring equals $m(1-m) \gamma \lambda z$. The expected cost of using the payment system has two components. There is the direct cost of using the system, $\delta x m(1-m)(1-\lambda) p$. There is also an indirect cost from trades that cannot take place because the payment system fails to function but that would have been executed if the buyer was carrying cash. This cost is $x m(1-m)(1-\delta)(1-\lambda)(u-c)$. 


\section{Conclusions}

In this paper we have introduced a payment system into a search theoretic model of money and examined its implications for social welfare. The model we use is that of $\mathrm{He}$ et al (2003). They present a model where money is subject to the threat of theft. They then introduce banks into the model. Agents can deposit their money in a bank and are issued with a cheque book or a debit card. These cannot be stolen. They show that cheques/debit cards may be necessary for there to be a monetary equilibrium. If there banks there must also be an inter-bank payment system through which banks can make transfers to each other. He et al assume that this system is risk-free and hence, largely subsume its role in their model. But in the real world payment systems are not risk free. In particular, they can suffer from two risks: credit risk and operational risk. Credit risk arises when there is a lag between when a producer receives a payment, say a cheque, and when the cheque is settled. A producer is in effect extending credit to a buyer until the cheque is settled. But during the lag the buyer's bank may default on its payment obligations. Lester (2004) has analysed the impact of this risk on agents' incentives to use the payment system. In this paper we have focused on operational risk. In payment systems that depend on the sending of electronic messages, there is the risk that the systems temporarily fail to function for some exogenous technological reason and prevent the system from being used.

We introduced operational risk into the He et al model by assuming that operational problems that prevent the payment system was working arrive according to a Poisson process. Agents do not know whether the system will function or not when they choose whether to use the payment system rather than carry money on their person. We show that agents have an incentive to use the payment system if it is both sufficiently cheap and sufficiently reliable. Agents have an incentive to use the system if or if not money is an accepted medium of exchange in the absence of a payment system. We then proceeded to consider the implications for social welfare of introducing a payment system. If the is not a monetary equilibrium in the absence of a payment system, introducing a system will unambiguously improve social welfare because it leads to trade occurring. When there is a monetary equilibrium in the absence of a payment system, social welfare may increase or decrease. It increases if the cost saving from thieves no longer incurring the cost they incur each time they successfully rob someone is greater than the costs from using the payment system. 


\section{References}

He, P, Huang, L, and Wright, R (2003), 'Money and banking in search equilibrium', University of Pennsylvania, mimeo.

Lagos, R, and Wright, R (2002), 'A unified framework for monetary theory and policy evaluation', Penn. Institute for Economic Research Working Paper, No.40.

Lester, B (2004), 'Payment systems in search equilibrium with bank default', Bank of England, mimeo.

Padoa-Schioppa, T (2002), 'Central banks and financial stability: Exploring the land in between', presented at the $2^{\text {nd }}$ ECB Central Banking Conference, Frankfurt, 24-25 October. 